
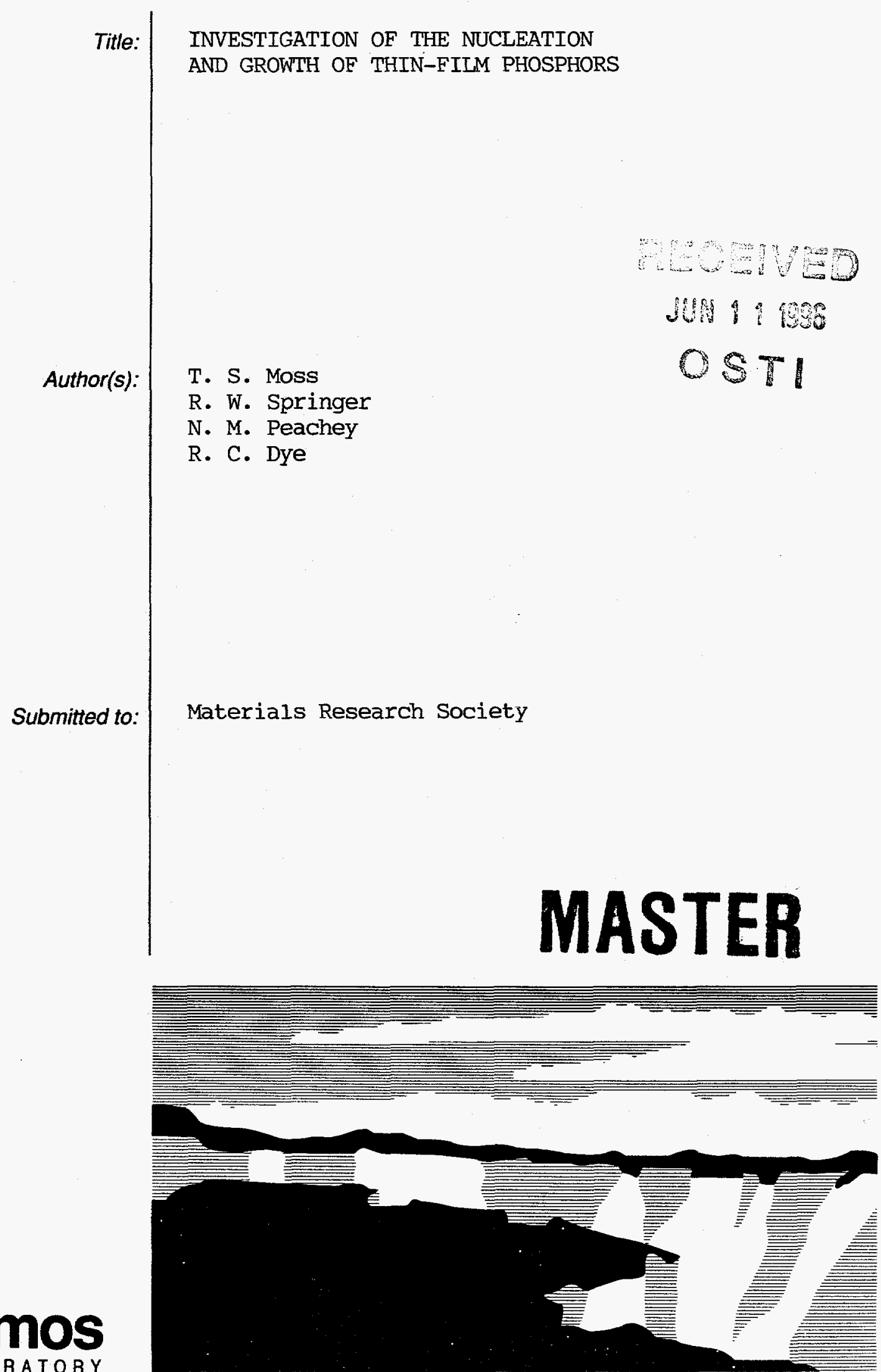

Los Alamos National Laboratory, an affirmative action/equal opportunity emplóyer, is operated by the University of California for the U.S. Department of Energy under contract $W$-7405-ENG-36. By acceptance of this article, the publisher recognizes that the U.S. Government retains a nonexclusive, royalty-free license to publish or reproduce the published form of this contribution, or to allow others to do so, for U.S. Government purposes. The Los Alamos National Laboratory requests that the publisher identify this article as work performed under the auspices of the U.S. Department of Energy. 


\section{DISCLAIMER}

Portions of this document may be illegible in electronic image products. Images are produced from the best available original document. 


\title{
LA-UR- $96-1448$
}

\section{INVESTIGATION OF THE NUCLEATION AND GROWTH OF THIN-FILM PHOSPHORS}

\author{
T.S. Moss, R.W. Springer, N.M. Peachey, and R.C. Dye
}

Los Alamos National Laboratory, M.S. E549, Los Alamos, NM 87545

\section{ABSTRACT}

The deposition of $\mathrm{CaGa}_{2} \mathrm{~S}_{4}: \mathrm{Ce}$ has been accomplished using a commercial liquid delivery system on two substrate surfaces: $\mathrm{ZnS}$ and SrS. However, the film deposited on $\mathrm{ZnS}$ was not of satisfactory quality because of the formation of an amorphous layer and a high amount of residual porosity within the deposition. The use of a SrS layer on top of the $\mathrm{ZnS}$ improved the nucleation by reducing the interfacial energy between the substrate and deposition. It greatly reduced the porosity in the coating and reduced the formation of the amorphous layer. The crystallinity of the $\mathrm{CaGa}_{2} \mathrm{~S}_{4} 400$ peak was also increased by a factor of ten when a layer of $\mathrm{SrS}$ was used. Further, the FWHM of the 400 peaks from the two depositions was not significantly different, indicating that the crystallite size and strain was approximately the same. The $\mathrm{B}_{40}$ was increased by a factor of two, from $1.84 \mathrm{~cd} / \mathrm{m}^{2}$ for $\mathrm{ZnS}$ to $3.67 \mathrm{~cd} / \mathrm{m}^{2}$ for $\mathrm{SrS}$. This increase is an improvement in the performance of the films and is attributable to the increase in the crystallinity.

\section{INTRODUCTION}

The development of an efficient blue phosphor for use in thin film electroluminescent displays (TFEL) is an important milestone in their deployment. It has been recently reported that $\mathrm{Ce}^{+3}$ doped alkaline earth thiogallate materials show emission with chromaticities within the blue region[1]. Cerium-doped calcium thiogallate $\left(\mathrm{CaGa}_{2} \mathrm{~S}_{4}: \mathrm{Ce}\right)$ is such a material whose production has been and continues to be actively pursued. In the recent past, a full color VGA TFEL panel has been fabricated and tested[1]. The deposition of $\mathrm{CaGa}_{2} \mathrm{~S}_{4}: \mathrm{Ce}$ has been reported from several methods, including sputtering[1], molecular beam epitaxy[2], and multisource deposition[3]. However, problems inherent to these techniques have prevented their widespread adaptation to a manufacturing scale. The primary limitation has been in the required extra annealing step to produce the crystallographic properties necessary for optimum luminance. The extra processing step also limits glass substrates to the high temperature compositions, adding to the overall cost of the display.

The process of metal-organic chemical vapor deposition (MOCVD) is well suited to overcome the limitations of the other techniques because it is capable of rapidly producing high quality, crystalline material at temperatures below $600^{\circ} \mathrm{C}$. The relatively simple deposition equipment is easily integrated into a manufacturing process, and several of the layers necessary for the TFEL device may also be produced using MOCVD, further simplifying the production scheme.

The MOCVD of $\mathrm{CaGa}_{2} \mathrm{~S}_{4}$ :Ce has been previously reported by this group[4-7]. These papers have dealt with the deposition using two reagent systems: traditional thermal bubblers and a commercial liquid delivery system. However, analyses of the electroluminescent (EL) properties of samples produced from these two techniques have not produced coatings with optimal EL emissions. Further studies of the samples have shown that the nucleation and growth characteristics have not been producing the expected dense films. As a result, the processes influencing the nucleation of $\mathrm{CaGa}_{2} \mathrm{~S}_{4}: \mathrm{Ce}$ have been investigated with the intent of better understanding how to positively influence the EL properties.

\section{EXPERIMENTAL}

The deposition of the $\mathrm{CaGa}_{2} \mathrm{~S}_{4}: \mathrm{Ce}$ phase was done using mixtures of $\mathrm{Ca}(\mathrm{tmhd})_{2}$, $\mathrm{Ga}$ (tmhd) $)_{3}, \mathrm{Ce}(\mathrm{tmhd})_{4}$, and $\mathrm{H}_{2} \mathrm{~S}$. The reagent delivery was performed using a liquid delivery system from Advanced Technology Materials, Inc., model LDS-300B. A description of the reagent delivery process has been described elsewhere[7]. Depositions were done at a temperature of $582{ }^{\circ} \mathrm{C}$ and a pressure of 5 torr. The substrates were glass slides with deposited conductive, 
dielectric, and nucleation layers and were furnished by Planar Systems, Inc. In this study, two nucleation layers were used: $\mathrm{ZnS}$ and $\mathrm{SrS}$. In the case of SrS surfaces, the SrS was deposited on top of the $\mathrm{ZnS}$. This arrangement could be completed into a device by the subsequent deposition of a top dielectric layer and metallic contact and permitted EL measurements to be made on the asdeposited material.

Samples were characterized for chemical composition, and microstructure by $\mathrm{x}$-ray fluorescence (XRF), x-ray diffraction (XRD), and transmission electron microscopy (TEM). Where possible, EL spectra were taken to determine the color and brightness of the emission. XRF was done on a Tracor unit, and XRD measurements were taken on a rotating anode Rigaku diffractometer using a position sensitive detector and an incident angle of $20^{\circ}$. SEM was done on a Hitachi S-4200, and a JEOL 2010 was used for TEM micrographs of sample cross-sections.

\section{RESULTS AND DISCUSSION}

The initial work on the deposition of the $\mathrm{CaGa}_{2} \mathrm{~S}_{4}: \mathrm{Ce}$ was accomplished using the $\mathrm{ZnS}$ deposition surface. The $\mathrm{ZnS}$ surface was considered to be desirable as a nucleation surface for $\mathrm{CaGa}_{2} \mathrm{~S}_{4}: \mathrm{Ce}$, and prior experience showed that its presence increased the EL brightness. However, an examination of the quality of material that was produced when the ZnS surface was used shows that the deposition was far from ideal. An example of the problems is illustrated in a cross-sectional TEM micrograph of deposited $\mathrm{CaGa}_{2} \mathrm{~S}_{4}: \mathrm{Ce}$, shown in Figure 1. This micrograph shows that the grains are long and thin with a width of 50 to $80 \mathrm{~nm}$. The grains appear to emanate from an interface with no evidence of renucleation. A closer inspection of the deposition grown near the interface showed that there was a narrow nucleation zone with little competitive growth between grains near the interface. Also in this micrograph, it is possible to see that there is a large amount of included porosity. The location of this porosity appears to be within the deposition and on top of an amorphous looking layer, as if the crystalline material had renucleated on top of an amorphous $\mathrm{CaGa}_{2} \mathrm{~S}_{4}: \mathrm{Ce}$ layer.

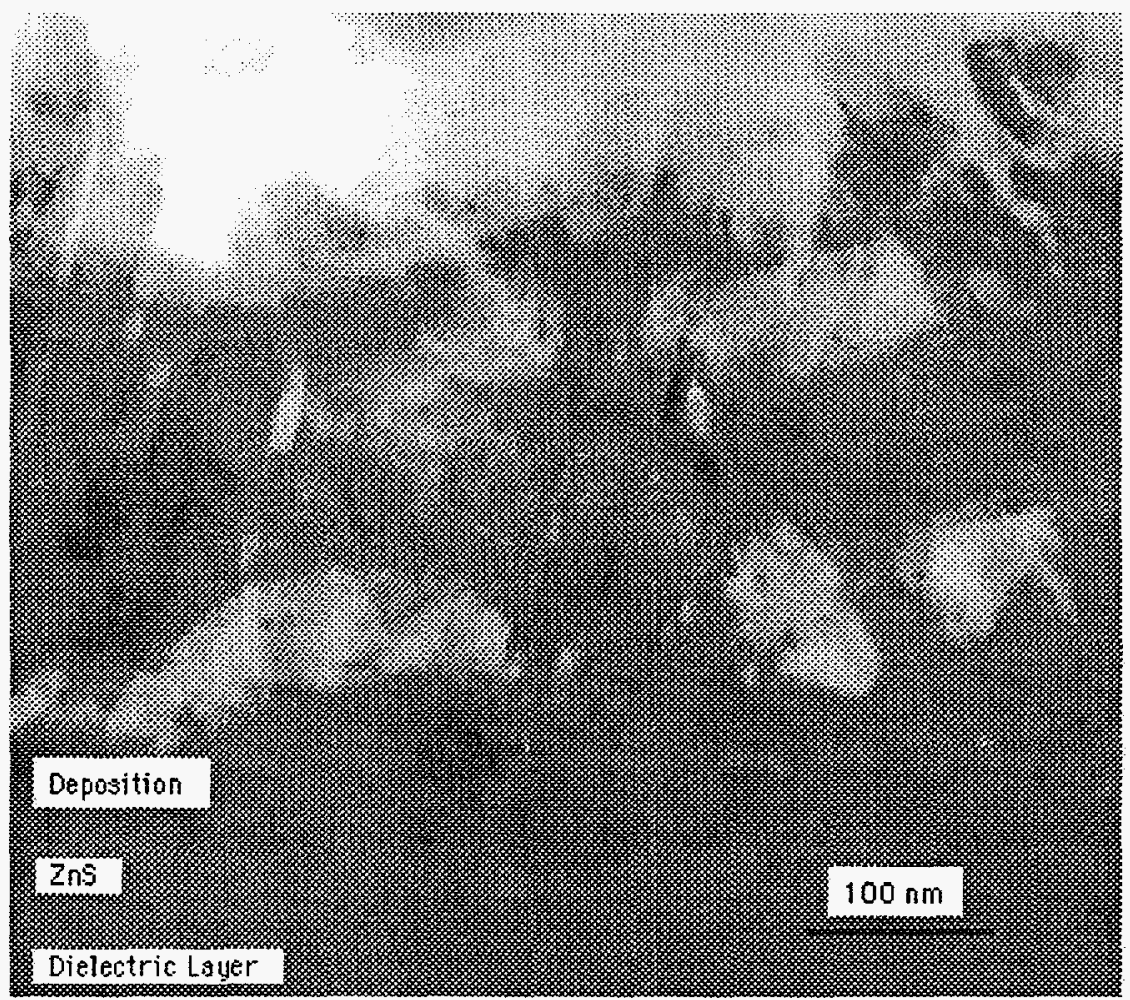

Figure 1. The deposition onto $\mathrm{ZnS}$ surfaces has included porosity within the deposition. 
To clarify some of the issues surrounding the quality of the initial layer, a second sample was prepared for TEM from a sample with a deposition time of 2.5 minutes, as opposed to 20 minutes. This micrograph is shown in Figure 2. From this picture, it is possible to observe the initial nuclei that formed on the surface of the $\mathrm{ZnS}$. They appear as dark, rectangular areas, growing away from the $\mathrm{ZnS}$ and have a width of approximately $10 \mathrm{~nm}$ and a height of $40 \mathrm{~nm}$. They appear to be crystalline by virtue of their uniform dark color which lighten when tilting the sample. The density of the nuclei on the surface appears to be quite low (on the order of 1 every $100 \mathrm{~nm}$ as observed on the lower magnification pictures, not shown here). Moire fringes also are present in an area near the center of the micrograph, as well as two other areas which are not as clearly visible. This would indicate that there are crystalline grains within the deposition. However, the rest of the film has an amorphous texture to it. The diffraction pattern of the entire coating did not show any defined spots or rings; further indicating that the bulk of the initial deposition was amorphous.

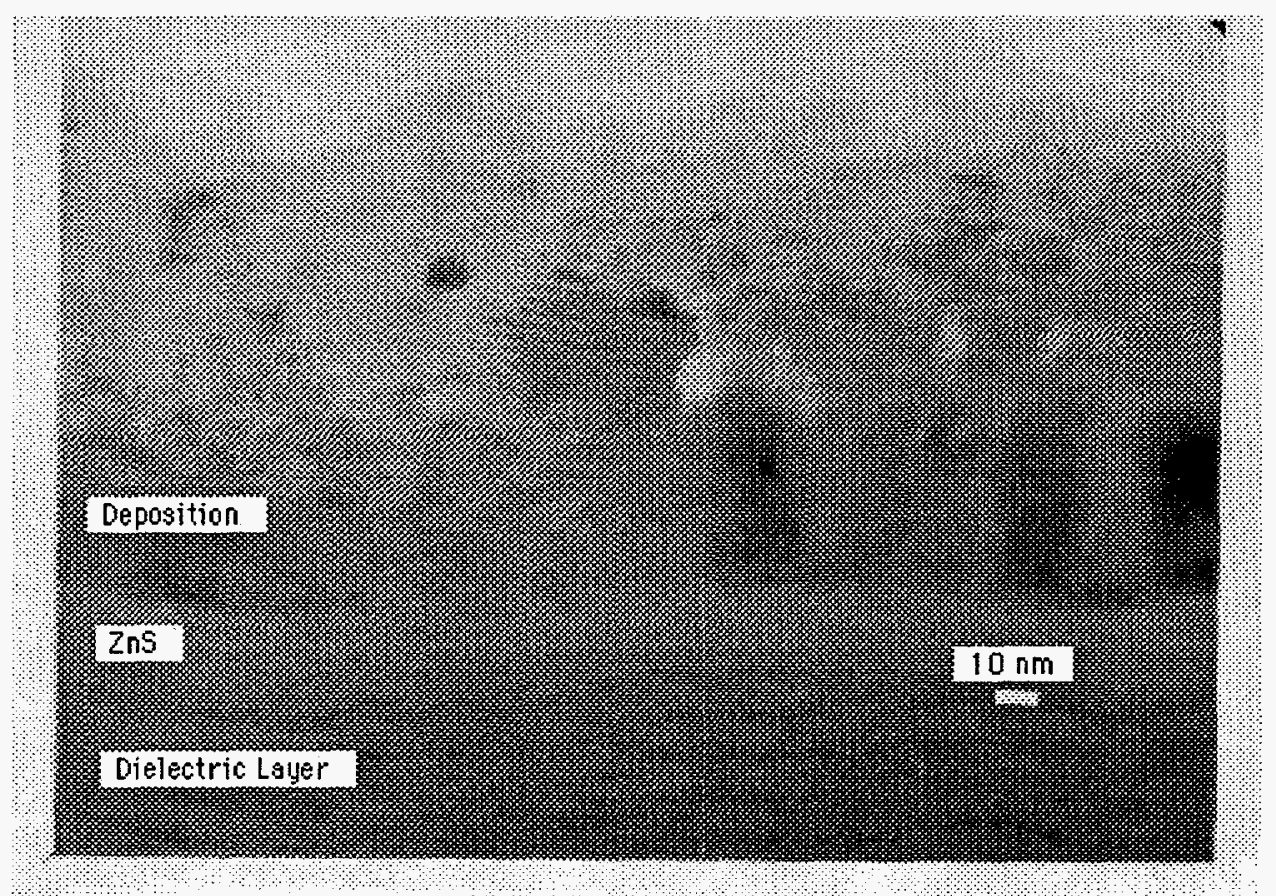

\section{Figure 2. Cross-section TEM of initial deposition shows an amorphous layer with small crystallites.}

It is possible to speculate that the deposition is occurring when an initial layer composed of a mixture of small crystalline grains and amorphous material. At some point after this layer is deposited, a second layer is nucleated, containing the large and highly crystalline grains which grow for the duration of the experiment. The nucleation of this second layer results in large amounts of porosity because the growth rate is so much faster than the nucleation rate. This type of a nucleation and growth mechanism is not an ideal situation for the deposition of high quality materials because of the high amounts of amorphous material and included porosity. Among the easier ways to alter the nucleation mechanism is to change the deposition surface such that the interfacial energy between the nucleus and the surface is reduced. If the interfacial energy is lowered by a different substrate surface, the nucleation rate is expected to increase because the critical radius to form a nucleus will decrease.

The choice for a new substrate surface was SrS. It was anticipated that the SrS would provide a better nucleation surface by the reduction of the interfacial energy. Further, if a boundary layer was formed between the substrate and the coating, SrS would improve the EL properties. In the case of the $\mathrm{ZnS}$, the boundary layer would be either $\mathrm{Zn}_{\mathrm{x}} \mathrm{Ca}_{1-\mathrm{x}} \mathrm{Ga}_{2} \mathrm{~S}_{4}: \mathrm{Ce}$ or $\mathrm{ZnGa}_{2} \mathrm{~S}_{4}: \mathrm{Ce}$; neither of these compounds would improve the luminance of the film and could shift the EL color towards the green. The use of $\mathrm{SrS}$ would form either $\mathrm{Sr}_{x} \mathrm{Ca}_{1-x} \mathrm{Ga}_{2} \mathrm{~S}_{4}: \mathrm{Ce}$ or 
$\mathrm{SrGa}_{2} \mathrm{~S}_{4}: \mathrm{Ce}$ as a boundary layer. These compounds have the added benefit of having a blue or a blue-green emission and are also fairly bright.

The change in the nucleation behavior was quite dramatic when the SrS substrates were used. A TEM cross-section is shown in Figure 3. This figure shows a sample which was deposited using the same deposition conditions as the sample seen in Figure 1. The width of the grains of this sample ranged from 75 to $125 \mathrm{~nm}$, an increase above that seen from the $\mathrm{ZnS}$ substrate. Also, it is worth noting that there appears to be a wider distribution of the grain sizes with the SrS substrate, indicating that there is a high amount of competitive grain growth. The increase in the degree of competitive growth would be expected from a sample with a higher nucleation rate because the increased number of nuclei must vie for space to grow into with greater frequency. Of particular interest is the reduced amount of porosity which is seen in the film. This observation is attributed to the reduced interfacial energy between the nucleus and the substrate which led to an increased nucleation rate. Further, there does not appear to be the large amount of amorphous deposition which was observed in the deposition onto $\mathrm{ZnS}$

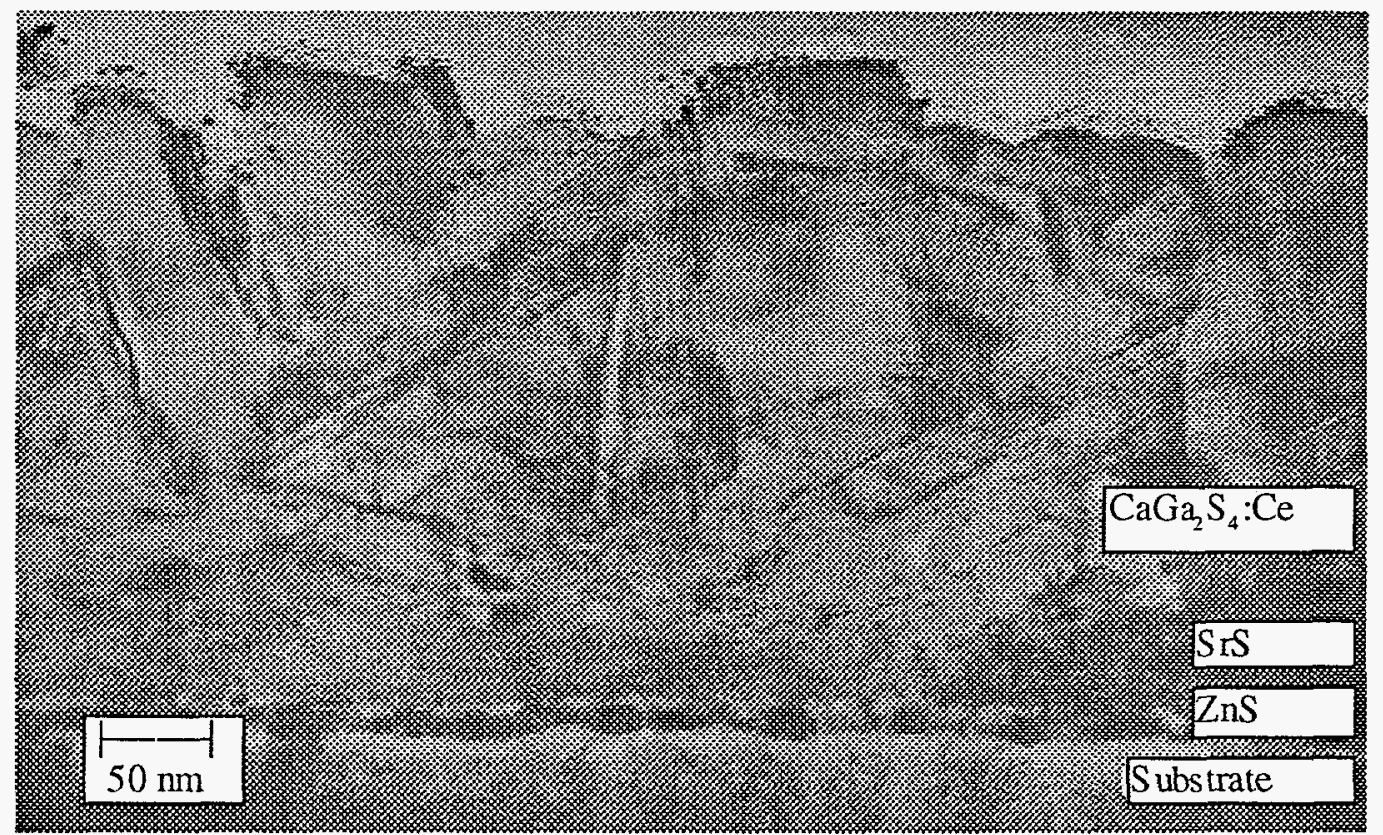

Figure 3. The use of $\mathrm{SrS}$ as the deposition surface greatly reduced the amount of porosity in the coating.

The use of SrS substrates also affected the crystallinity of the deposition. The 400 peak in the $\mathrm{CaGa}_{2} \mathrm{~S}_{4}$ pattern (JCPDS Card 25-134) was used to gauge the crystallinity since it was present in most every pattem when $\mathrm{CaGa}_{2} \mathrm{~S}_{4}$ was deposited. Because the patterns were taken with different sampling times, the count rate rather than the peak height is of interest. The count rate for the 400 peak for the sample deposited on $\mathrm{ZnS}$ and shown in Figure 1 was 0.59 counts/second. This rate is compared with the SrS substrate shown in Figure 3 which had a count rate of 8.29 counts/second. Based on this difference, it is possible to determine that the use of SrS as the substrate surface either increased the crystallinity of the deposition or produced a material with much higher preferred orientation. However, taking into account the difference in the apparent porosity and reduced amount of amorphous material, it is probable that depositing onto SrS produced a sample with more crystalline grains.

The full-width, half maximum (FWHM) of the 400 peak is also of interest as to how the change in substrate surface affects the deposition process. Visual measurements of the peaks were made to determine there was a difference in the crystallite size or strain. While these measurements were made without taking in account the broadening due the diffractometer, they did serve as a qualitative technique to evaluate relative effects. The results show that there was no determinable difference in the FWHM of the 400 peak between the $\mathrm{ZnS}$ (FWHM=0.13 ${ }^{\circ} 2 \theta$ ) and the $\operatorname{SrS}$ 
(FWHM $=0.12^{\circ} 2 \theta$ ). The difference between the two values was not resolvable within the error of the diffractometer, indicating that the quality of the material deposited on the two surfaces was not determinably different.

The EL of these two samples was expected to show an increase in the brightness which would correspond to the increased crystallinity. The brightness of the deposition on $\mathrm{ZnS}$ at 40 volts above the turn-on voltage $\left(B_{40}\right)$ was $1.84 \mathrm{~cd} / \mathrm{m}^{2} ; B_{40}$ for the deposition on $\mathrm{SrS}$ was 3.67 $\mathrm{cd} / \mathrm{m}^{2}$. The result is roughly a factor of two improvement when using the SrS surface. This increase in the brightness was welcomed as an improvement to the deposition and was attributed to the increase in the crystallinity, as evidenced by the changes in the $400 \mathrm{CaGa}_{2} \mathrm{~S}_{4}$ peak. Further increases in the brightness are expected to come from optimizing the Ce doping and minimizing the amount of $\mathrm{Ga}_{2} \mathrm{~S}_{3}$ in the deposition. However, because the $\mathrm{SrS}$ substrates were layered on the borosilicate glass, the XRF measurements of the samples have not been able to accurately determine the quantity of $\mathrm{Ca}$ and $\mathrm{Ce}$ in the films due to peak overlap with elements contained in the glass. As a result, the degree of Ce doping could not be accurately resolved nor could the amount of $\mathrm{Ga}_{2} \mathrm{~S}_{3}$ be estimated. Both the Ce doping and the $\mathrm{Ga}_{2} \mathrm{~S}_{3}$ could affect the value of $\mathrm{B}_{40}$. Changing to a different composition glass or a different analytical technique should alleviate these problems.

\section{CONCLUSIONS}

The deposition of $\mathrm{CaGa}_{2} \mathrm{~S}_{4}$ : $\mathrm{Ce}$ has been accomplished using a commercial liquid delivery system. However, the deposition onto $\mathrm{ZnS}$ surfaces was not ideal because of problems in the nucleation and growth of the film. The deposit was defined by two apparent layers: an amorphous initial layer and a renucleated crystalline layer. A closer observation of the initial layer showed some small areas of crystalline grains, including several nuclei, but the bulk of the coating was amorphous in appearance. A high amount of this porosity was observed at the interface between the two layers. This indicated that the second layer grew at a much higher rate than it nucleated. A change to SrS resulted in a continuous coating with greatly reduced porosity and increased crystallinity. The crystallinity of the $\mathrm{CaGa}_{2} \mathrm{~S}_{4}$ on $\mathrm{SrS}$ was also increased by a factor of over ten based on the 400 peak. The FWHM of the 400 peaks between the two substrates was not significantly different, indicating that the amount of crystallite size and strain was about the same. The $\mathrm{B}_{40}$ was increased by a factor of two, from $1.84 \mathrm{~cd} / \mathrm{m}^{2}$ for $\mathrm{ZnS}$ to $3.67 \mathrm{~cd} / \mathrm{m}^{2}$ for SrS. This increase in the brightness is an improvement in the performance of the films and is attributable to the increase in the crystallinity.

\section{ACKNOWLEDGMENTS}

We wish to acknowledge the work of Chris Schaus and Mark DelaRosa at Planar Systems, Inc., for providing the substrates and EL measurements and the work of Ms. Kerry Siebein for her work in providing several of the TEM micrographs.

\section{REFERENCES}

1. W.A. Barrow, R.C. Coovert, E. Dickey, C.N. King, S. Sun, R.T. Tuenge, R. Wentos, and J. Kane, S.I.D. 93 Digest, 24, 761 (1993).

2. K. Tanaka, Y. Inoue, S. Okamoto, and K. Kobayashi, J. Cryst. Growth, 63, 1954 (1995).

3. T.A. Oberacker, K.O. Velthaus, R.H. Mauch, H.W. Stock, and R.T. Tunge, 1994 Workshop on Electroluminescent Displays, Digest of Technical Papers, (1994).

4. N.M. Peachey, J.A. Samuels, B.F. Espinoza, C.D. Adams, D.C. Smith, R.C. Dye, R.T. Tuenge, C.F. Schaus, and C.N. King in Novel Techniques in Synthesis and Processing of Advanced Materials, edited by J. Singh and S.M. Copley (The Minerals, Metals \& Materials Society, 1995), p. 365.

5. T.S. Moss, J.A. Samuels, D.C. Smith, R.C. Dye, M.J. DelaRosa, and C.F. Schaus in Diversity into the Next Century, edited by R.J. Martinez, H. Arris, J.A. Emerson, and G. Pike (Society for the Advancement of Materials and Processes Engineering, Covina, CA, 1995),.27, p. 507 , 
6. T.S. Moss, D.C. Smith, J.A. Samuels, R.C. Dye, M.J. DelaRosa, and C.F. Schaus, submitted to S.I.D. Digest (1995).

7. T.S. Moss, R.C. Dye, D.C. Smith, J.A. Samuels, M.J. DelaRosa, and C.F. Schaus, accepted for MOCVD of Electronic Ceramics II, Materials Research Society (1995).

\section{DISCLAIMER}

This report was prepared as an account of work sponsored by an agency of the United States Government. Neither the United States Government nor any agency thereof, nor any of their employees, makes any warranty, express or implied, or assumes any legal liability or responsibility for the accuracy, completeness, or usefulness of any information, apparatus, product, or process disclosed, or represents that its use would not infringe privately owned rights. Reference herein to any specific commercial product, process, or service by trade name, trademark, manufacturer, or otherwise does not necessarily constitute or imply its endorsement, recommendation, or favoring by the United States Government or any agency thereof. The views and opinions of authors expressed herein do not necessarily state or reflect those of the United States Government or any agency thereof. 